\title{
Efficacy of Transosseous Tunnel Placement for Double Endobutton Platet in the Distal Tibiofibular Syndesmosis Reconstruction :A Three-Dimensional Printing Guide Design Technology
}

\section{Lei Zhang}

The Affiliated Traditional Chinese Medicine Hospital of Southwest Medical University

Jixiang Xiong

Southwest Medical University

Xin Zhou

The Affiliated Traditional Chinese Medicine Hospital of Southwest Medical University

\section{Xiangyu Tang}

Southwest Medical University

\section{Yiwen Gan}

Southwest Medical University

\section{Yuening Yang}

Southwest Medical University

\section{Zhangrong Xia}

Southwest Medical University

\section{Xinghao Sun}

Southwest Medical University

Tianyu Liu

Southwest Medical University

Guoyou Wang ( $\nabla$ wang_guoyou1981@163.com )

The Affiliated Traditional Chinese Medicine Hospital of Southwest Medical University

\section{Research Article}

Keywords: Transosseous Tunnel Placement, the distal tibiofibular syndesmosis reconstruction, ThreeDimensional Printing, Guide Design

Posted Date: February 22nd, 2022

DOI: https://doi.org/10.21203/rs.3.rs-1311277/v1 
License: (c) (i) This work is licensed under a Creative Commons Attribution 4.0 International License. Read Full License 


\section{Abstract \\ Background}

To search for a method of accurate transosseous tunnel drilling for the distal tibiofibular syndesmosis based on three-dimensional (3D) printing technology to design a guide design and to assess its veracity.

\section{Methods}

CT scan and MRI data of 150 patients with distal tibiofibular syndesmosis injury in the Affiliated Hospital of traditional Chinese medicine of Southwest Medical University were selected. Using Mimics software to reconstruct inferior tibiofibular syndesmosis CT data. Design 2 The non-collinear tunnel is superimposed on the three-dimensional model, and a virtual drilling is performed between the tibia and the fibula using a double inner gusset. Then, in the Geomagic Studio software model, an elliptical plane is calculated and extracted as a guide design for precise drilling. Then put the design and the 3D inferior tibiofibular syndesmosis model together for 3D printing. 8 parameters were measured, and the effects of the virtual model and the actual model and the guide rail design were compared.

\section{Results}

We compared 8 parameters of the 3D virtual model and actual model successfully. No differences were significantly found between the real and virtual bone tunnels in measurements. $(P>0.05)$.

\section{Conclusions}

The veracity of transosseous tunnel placement for distal tibiofibular syndesmosis reconstruction combined with 3D printing guide design technology in the distal tibiofibular syndesmosis is reliable.

\section{Introduction}

The distal tibiofibular syndesmosis is a kind of fretting elastic joint, which is composed of distal tibia ,distal fibula and lower tibiofibular ligament complex [1]. It has effect of stabling ankle joint and is one of the common injuries in orthopedic and sports medicine field. The incidence is $1 \%-11 \%[2-4]$ and it is increasing progressively year by year. we know that anatomical reconstruction and surgical fixation of inferior tibiofibular syndesmosis are key to achieving the best results, and misdiagnosis or improper management of combined injuries can lead to lateral instability of the ankle(LIA) accompanied by persistent ankle pain, early traumatic arthritis, and ultimately changes in ankle arthritis [6-8, 23]. Nowadays, many scholars have been studying and discussing the structure, function and diagnosis and treatment on it. The research focuses on improving or developing surgical reconstruction method or internal fixation device which is more suitable for the biomechanical characteristics of it. By many 
literature studies, the best effect of combined reconstruction of the distal tibiofibular syndesmosis is Endobutton plate fixation technique [9-12, 22]. The distal tibiofibular syndesmosis reconstruction by Endobutton plate fixation is reported in many articles. The operation is performed at $1 \sim 2 \mathrm{~cm}$ of the ankle, from the posterior to the anterior, through the distal tibiofibular syndesmosis and the ankle horizontally into $20^{\circ} \sim 30^{\circ}$ angles, drilled through the fibula plate with a $2.0 \mathrm{~mm}$ guide needle and elicited from the medial tibial skin [10]. By Henry DeGroot et al. study[12], the device is easy to use and can allow load-bearing without fear of implant breakage. Besides, it leaves physiological fretting function without secondary surgical removal, implant fatigue. However, it is worth noting that although Endobutton plate fixation technology has solved many problems, there are still some shortcomings, the length of loop during operation can not be adjusted at will, and complications such as wound infection, local stimulation, screw loosening or fracture may occur. For solving these problems, many scholars try to use $3 \mathrm{D}$ of print technology Combined treatment of inferior tibiofibular joint.To solve these problems, we tried to determine the Transosseous Tunnel Placement for Double Endobutton platet in the distal tibiofibular syndesmosis reconstruction.

Hence in our study, we compare the difference between the actual model and the and 3D virtual model, reconstruct the dital tibiofibular syndesmosis by using doeble Endobutton plate fixation technique under the aid of $3 \mathrm{D}$ print-oriented design of Transosseous Tunnel Placement. The aim is to find a novel internal fixation device for the reconstruction of the dital tibiofibular syndesmosis without complications. furthermore, this study verified the feasibility, safety and accuracy of the $3 \mathrm{D}$ print-guided design of bone marrow channel navigation module, and provided theoretical support and experimental basis for its clinical assisted reconstruction of tibiofibular syndesmosis.

\section{Materials And Methods}

\section{Ethical statement}

All procedures were allowed by the Medical Ethics Review Board of Affiliated Traditional Chinese Medicine Hospital of Southwest Medical University with the following reference number: KY2018043.

\section{Patients}

We collected 150 patuents computed tomography (CT) plain scan and the MRI data of lower tibia and fibula from the radiology department of the affiliated traditional Chinese medicine hospital of Southwest Medical University. Exclusion criteria included congenital malformations and Fracture of tibia and fibula.We selected 100 neat and complete inferior tibiofibular syndesmosis (50 on the left and 50 on the right) from 150 cases for digital imaging and medical communication (DICOM) data.

\section{The design of 3D printing guide design}

The scanning data CT tibia and fibula and all the data were imported into the image processing software (Mimics19.0), and the virtual 3D models wre constructed. Besides,the tibia and fibula to test and drill 
accurately had been preserved. Based on the anatomical measurements of the $\mathrm{CT}$, the optimal drilling points on the tibia and fibula were measured, followed by the use of 2 noncollinear hollow cylinders (inner radius $2 \mathrm{~mm}$ and upper half diameter $4 \mathrm{~mm}$ ). The superposition on the 3D model represented the virtual drilling between the tibia and fibula. At the same time, the 3D model were imported into the software (Geomagic Studio2013. 0) to get the oval model was calculated and extracted along the plane around the clavicle orifice (thickness $2.5 \mathrm{~mm}$, direction upward) as a guiding design for accurate borehole reconstruction of inferior tibiofibular joint during surgery. combining design and 3D models in Mimics17.0. Maker Bot printing software was used to convert the combined data into the printer Z18 printer printing file (printing parameters: printing mode, balance; layer height $0.2 \mathrm{~mm}$; wall thickness 2 times) nozzle thickness; nozzle moving speed , $150 \mathrm{~mm} / \mathrm{s}$; nozzle temperature ,215 nozzle wire diameter , $1.77 \mathrm{~mm}$; platform Withdrawal height $0.5 \mathrm{~mm}$; bottom thickness $0.8 \mathrm{~mm}$, top thickness $0.804 \mathrm{~mm}$; minimum support angle 68 degrees, support density $16 \%$, printing material: biodegradable plastic polylactic acid).

\section{The parameters of 3D printing guide design}

At $1 \sim 2 \mathrm{~cm}$ above the fibular ankle joint space, from the back to the front and in, $20^{\circ} \sim 30^{\circ}$ to the articular surface. The bone tunnel was established obliquely through the inferior tibiofibular syndesmosis. Reference point a: the center of tibial tunnel. Reference point $b$ : the center of the sural tunnel.

The virtual tunnel can be measured recorded with an accuracy of up to $0.1 \mathrm{~mm}$ in Mimics 19.0. To avoid observer variation, an investigator with over 3 years of experience in 3D printing work to measure and record 8 parameters carefully 3 times,and then take the average. (Fig1,2,3,4)

1. ac: The distance from the center of tibial tunnel to the proximal end of medial malleolus.

2. ad: The distance from the center of the lateral tibial tunnel to the anterior tibia.

3. ae: The distance from the center of the tibial tunnel to the posterior tibia.

4. bf: The distance from the center of fibular tunnel to the proximal end of lateral malleolus.

5. bg: The distance from the center of the lateral fibular tunnel to the anterior fibula.

6. bh: The distance from the center of the lateral fibular tunnel to the posterior fibula.

7. L: Length of bone marrow canal.

8. $a \rrbracket$ Angle between bone marrow canal and horizontal plane.

\section{Statistical analysis}

Measurement data were expressed as mean \pm standard deviation $( \pm s)$ in $\mathrm{mm}$. A paired two-tailed t-test was performed to identify differences between measurements in 3D virtual model and actual model if the data were normally distributed. Statistical analysis was performed using the SPSS26.0 (IBM Corp., Armonk, NY, USA); $P<0.05$ was considered statistically significant. (Table 1 )

\section{Results}


We have printed 150 human inferior tibiofibular syndesmosis successfully. Then 8 parameters of the 3D virtual model and actual model with the guide design were measured and compared in Figure 1,2. We found that there was no difference between the actual and virtual bone tunnels in ac, ad, ae, bf, bg, bh, $L$, a, $\beta(P>0.05)$. All data were represented as $\pm s$ in Table 1.

\section{Discussion}

The distal tibiofibular syndesmosis is an important joint to stabilize the ankle. Alao it is a common clinical injury, accounting for $5-10 \%$ of ankle sprain and $23 \%$ of ankle fracture $[1,3,4]$. In the most cases, it is accompanied by other ankle injuries, which can easily cause lateral instability of the ankle(LIA) with long-term chronic pain and traumatic osteoarthritis, which seriously affects the quality of life and work of patients. At present, the treatment of the distal tibiofibular syndesmosis injury is a hot topic in our research. The main method of conservative treatment is to recover by functional exercise after 6-8 weeks fixed with gypsum or support, but for unstable lower tibia and fibula combined injury, surgical treatment is generally recommended. Nowdays, the widely applicable clinical surgical treatment includes rigid fixation (mainly cortical screw fixation) and elastic fixation (mainly Endobutton system)[9-10]. A few scholars have found that the incidence of broken nails is high due to its rigid fixation in the cortical screw fixation, patients can not early weight-bearing exercise, prone to ossification, ankle stiffness and traumatic arthritis, and often need secondary surgical removal, which increased the patient's mental trauma and financial burden, the risk of recurrence is high extremely [10,14,16], Mehdi et al .[15] experiments have proved that Significant screw bending occurred in severe patients with heavy weight after operation. taking these risks into account, some scholars have attempted to treat inferior tibiofibular joint injury by Endobutton plate fixation[8-10,16,18]. The Endobutton device is a novel inferior tibiofibular fixation device with a fixation strength close to the screw, and the semi-rigid biomechanical properties of the device allow physiological fretting between the lower tibia and fibula, thus accelerating ligament healing; no secondary surgery is required to reduce trauma $[3,19,21]$. Experiments show that there is no risk of screw breakage, no need for routine removal after surgery by Endobutton, and it can better restore ankle function, reduce related complications and other advantages, less trauma [ 16,20].

Although Endobutton plate fixation solves many clinical problems, some studies have reported that it also has some shortcomings. Lou YL et al .[10] research shows that the number of clinical surgical cases is too small at present, it is necessary to accumulate experience through the department operation practice, combined with relevant reports at home and abroad, and we can not guarantee the accuracy of the operation because of the problems of the angle of the needle entry, the position of the fixed device and the quantity of the device placed. As can be seen that double Endobutton the difficulty of double Endobutton plate fixation is the establishment of an ideal and safe and accurate transosseous tunnels . The length of the loop can not be adjusted at will in the operation. We must accurately determine the drill points on the tibia and fibula and the angle and size of the tunnel. in order to identify the ideal transosseous tunnels and coracoid process, many studies have investigated the anatomical features of the dital tibiofibular syndesmosis.But in fact, the location and method of drilling a transosseous tunnels in the tibia and fibula vary depending on the operator and the patient's skeletal structure can be 
affected.In that case, we combined the anatomical basis of the dital tibiofibular syndesmosis to study and design a personalized 3D printing guide design, placed on the exposed dital tibiofibular syndesmosis, to help find the precise anatomical location of the cross-bone tunnel borehole during surgery, and we experimentally compared the virtual $3 \mathrm{D}$ model with the double Endobutton plate fixation using the $3 \mathrm{D}$ navigation module to build the transosseous tunnels. From our experimental measurements, we found that there was almost no difference between the real model and the virtual model of Endobutton plate fixation. It can be seen that this method can improve the accuracy of transosseous tunnels and reduce the drilling accuracy risk of complications. So transosseous tunnels module based on the 3D printing guide design of the distal tibiofibular syndesmosis reconstruction of the is reliable and can be carried out.

\section{Limitations}

However, the following shortcomings also existed in our study :(1) this study only collected CT and MRI data from a hospital in southwest china, did not contain population data from multiple regions and between different races, and the subjects of the experiment were also patients in this hospital, which was not widely representative. (2) This study is an in vitro study and its rationality and availability are to be confirmed by further studies in clinical trials.

Our study has some weaknesses. First, the samples and individualized 3D printed guide design are imitated in this study. These prospective CT scans without considering whether the AC joint itself affect the building of the 3D guide design, which may lead to some observational errors. Finally, this study is an in vitro research, and its rationality and availability need to be confirmed by further studiess in clinical trials.

\section{Conclusion}

In conclusion, there is no difference between the 3D virtual model reconstructed by inferior tibiofibular syndesmosis and the actual model. The individualized 3D guide design for inferior tibiofibular syndesmosis can improve the accuracy of transosseous tunnels and decrease the risk of the complications. The accuracy of Inferior tibiofibular syndesmosis combined with 3D printing guidance design technology in the transosseous tunnel of distal tibiofibular syndesmosis reconstruction is reliable.

\section{Declarations}

\section{Statement}

All methods were carried out in accordance with relevant guidelines and regulations under Ethics approval and consent to participate. Informed consent was obtained from all subjects.

\section{Ethics approval and consent to participate}


All procedures were approved by the Ethical Committee of Affiliated Traditional Chinese Medicine Hospital of Southwest Medical University (No. KY2018043).

\section{Consent for publication}

All authors approved the final manuscript and consent for publication.

\section{Availability of data and materials}

The datasets used and analyzed during the current study are available from the corresponding author on reasonable request, and we are willing to share the research data after the article is published.

\section{Competing interests}

No conflicts interest existed in the submission of this manuscript, and the manuscript was approved by all authors for publication.

\section{Funding}

Health Commission of Sichuan Province Science and Research Project (Popularization and Application Project), grant number: 20PJ143. Luzhou People's Government-Southwest Medical University Shi-zhen Zhong Academician Talent Team Sub-project, grant number: 2018zszysrctdxm. Southwest Medical University Research Project, grant number囚2020ZRQNA045. Orthopedics (Shangantong) special scientific research project of Sichuan Medical Association, project number: 2020SAT26. Central Funds Guiding the Local Science and Technology Development General Program of Sichuan Provincial Science and Technology Department, project number:2021ZYD0078.

\section{Authors' contributions}

Lei Zhang and Guoyou Wang contributed to conception and design. Jixiang Xiong and Xin Zhou contributed to edit and process articles. Xiangyu Tang and Yiwen Gan contributed to data collection and picture data processing. Yuening Yang contributed to statistical analysis and picture data processing. Zhangrong Xia, Tianyu Liu and Xinghao Sun contributed to revise the manuscript.

\section{Acknowledgements}

The authors wanted to show their gratitude to the imaging department in Affiliated Traditional Chinese Medicine Hospital of Southwest Medical University that provided the samples.

\section{References}

1. Liu Zengjun, Li Jun, Meng Zhen. Clinical Anatomy of the Lower tibiofibular Combined Stabilization Complex [J]. Chinese Journal of Clinical Anatomy, 2011, 29(02): 162-164. 
2. Beumer A, van Hemert WL, Niesing R, et al. Radiographic measurement of the distal tibiofibular syndesmosis has limited use [J]. Clinical Orthopedics Related Research, 2004, 36(423): 227-234.

3. Cottom JM, Hyer CF, Philbin TM, et al. Transosseous fixation of the distal tibiofibular syndesmosis: comparison of an interosseous suture and endobutton to traditional screw fixation in 50 cases [J]. Journal of Foot and Ankle Surgery, 2009, 48(6): 620-630.

4. Tourné $Y$, Molinier $F$, Andrieu $M$, et al. Diagnosis and treatment of tibiofibular syndesmosis lesions [J]. Orthopaedics \& traumatology, surgery \& research: OTSR, 2019, 105(8): 275-286.

5. Liu GT, Ryan E, Gustafson E, et al. Three-Dimensional Computed Tomographic Characterization of Normal Anatomic Morphology and Variations of the Distal Tibiofibular Syndesmosis [J]. The Journal of foot and ankle surgery: official publication of the American College of Foot and Ankle Surgeons, 2018, 57(6): 1130-1136.

6. Sagi HC, Shah AR, Sanders RW. The functional consequence of syndesmotic joint malreduction at a minimum 2-year follow-up [J]. J Orthop Trauma, 2012, 26(7): 439-443.

7. Day GA, Swanson CE, Hulcombe BG. Operative treatment of ankle fractures: a minimum ten-year follow-up [J]. Foot Ankle Int, 2001, 22(2): 102-106.

8. van den Bekerom MP, de Leeuw PA, van Dijk CN. Delayed operative treatment of syndesmotic instability [J]. Curr Concepts Rev Inj, 2009, 40(11): 1137-1142.

9. Wang L, Wang B, Xu G, et al. Biomechanical comparison of bionic, screw and Endobutton fixation in the treatment of tibiofibular syndesmosis injuries [J]. Int Orthop, 2016, 40(2): 307-314.

10. Lou YL, Hong JJ, Shao XW, et al. Endobutton and cortical screw fixation for the treatment of distal tibiofibular syndesmosis separated [J]. Zhongguo Gu Shang, 2016, 29(8): 729-733.

11. Thornes B, Shannon F, Guiney AM, et al. Suture-button syndesmosis fixation: accelerated rehabilitation and improved outcomes [J]. Clin Orthop Relat Res, 2005, 431(431): 207-212.

12. Degroot $\mathrm{H}, \mathrm{Al}-\mathrm{Omari} \mathrm{AA}, \mathrm{El}$ Ghazaly SA. Outcomes of suture button repair of the distal tibio-fibular syndesmosis [J]. Foot Ankle Int, 2011, 32(3): 250-256.

13. Tsai J, Pivec R, Jauregui JJ, et al. Strength of Syndesmosis Fixation: Two TightRope versus One TightRope with Plate-and-Screw Construct [J]. Journal of long-term effects of medical implants, 2016, 26(2): 161-165.

14. Yan Ruijian, Zhang Xiaowen, Guo Qiufeng, et al. Clinical observation on the treatment of combined injury of lower tibia and fibula ligament with screw [J]. Bone injury in China, 2009, 22(11): 827-829.

15. Mehdi Mousavi, Alexander Egkher, Wolfgang Pichl, et al. Dynamics of Tibiofibular Syndesmosis in Correlation with the Level of the Syndesmotic Scress in Maisonneuvre Fractures of the Ankle - a Cadaver Study [J]. European Journal of Trauma, 2001, 27(2): 87-91.

16. Wu Shaokun, Yang Lei, Yan Sunfang, Liao Wei, et al. Treatment of simple inferior tibiofibular combined separation by Endobutton device [J]. Bone injury in China, 2012, 25(03): 224-227.

17. Forsythe K, Freedman KB, Stover MD, et al. Comparison of a novel Fiberwire-button construct versus metallic screw fixation in a syndesmotic injury model [J]. Foot Ankle Int, 2008, 29(1): 49-54. 
18. Lemmers DHL, Lubberts B, Stavenuiter R, et al. Factors associated with adverse events after distal tibiofibular syndesmosis fixation [J]. Injury, 2020, 51(2): 542-547.

19. Kurtoglu A, Kochai A, Inanmaz ME, et al. A comparison of double single suture-button fixation, suturebutton fixation, and screw fixation for ankle syndesmosis injury: A retrospective cohort study [J]. Medicine (Baltimore), 2021, 100(13): e25328.

20. Wang $\mathrm{L}$, Wang $\mathrm{B}, \mathrm{Xu} \mathrm{G}$, et al. Biomechanical comparison of bionic, screw and Endobutton fixation in the treatment of tibiofibular syndesmosis injuries [J]. Int Orthop, 2016, 40(2): 307-314.

21. Jia Z, Cheng J, Zhong H, et al. Titanium cable isotonic annular fixation system for the treatment of distal tibiofibular syndesmosis injury [J]. American Journal of Translational Research, 2019, 11(8): 4967-4975.

22. Fan X, Zheng P, Zhang Y, et al. Dynamic Fixation versus Static Fixation in Treatment Effectiveness and Safety for Distal Tibiofibular Syndesmosis Injuries: A Systematic Review and Meta-Analysis [J]. Orthopaedic Surgery, 2019, 11(6): 923-931.

23. Cornu O, Manon J, Tribak K, et al. Traumatic injuries of the distal tibiofibular syndesmosis [J]. Orthopaedics \& Traumatology Surgery \& Research, 2020, 107(1S): 102778.

\section{Tables}

Table 1: 8 parameters of the 3D virtual model and actual model with the guide design. 


\begin{tabular}{|c|c|c|c|}
\hline & Group & Longs & \\
\hline \multirow[t]{2}{*}{ ac } & actual model & 3.350 & $P>0.05$ \\
\hline & 3D virtual model & 3.278 & \\
\hline \multirow[t]{2}{*}{ ad } & actual model & 1.568 & $P>0.05$ \\
\hline & $3 \mathrm{D}$ virtual model & 1.574 & \\
\hline \multirow[t]{2}{*}{ ae } & actual model & 1.612 & $P>0.05$ \\
\hline & $3 \mathrm{D}$ virtual model & 1.620 & \\
\hline \multirow[t]{2}{*}{ bf } & actual model & 3.788 & $P>0.05$ \\
\hline & $3 \mathrm{D}$ virtual model & 3.772 & \\
\hline \multirow[t]{2}{*}{ bg } & actual model & 0.717 & $P>0.05$ \\
\hline & $3 \mathrm{D}$ virtual model & 0.722 & \\
\hline \multirow[t]{2}{*}{ bh } & actual model & 0.746 & $P>0.05$ \\
\hline & $3 \mathrm{D}$ virtual model & 0.745 & \\
\hline \multirow[t]{2}{*}{ L } & actual model & 4.940 & $P>0.05$ \\
\hline & 3D virtual model & 5.025 & \\
\hline \multirow[t]{2}{*}{$a$} & actual model & 27.9 & $P>0.05$ \\
\hline & 3D virtual model & 27.9 & \\
\hline
\end{tabular}

ac: The distance from the center of tibial tunnel to the proximal end of medial malleolus. ad: The distance from the center of the lateral tibial tunnel to the anterior tibia. ae: The distance from the center of the tibial tunnel to the posterior tibia.

bf: The distance from the center of fibular tunnel to the proximal end of lateral malleolus. bg: The distance from the center of the lateral fibular tunnel to the anterior fibula. bh: The distance from the center of the lateral fibular tunnel to the posterior fibula.

L: Length of bone marrow canal.

$\mathrm{a} \rrbracket$ Angle between bone marrow canal and horizontal plane. 


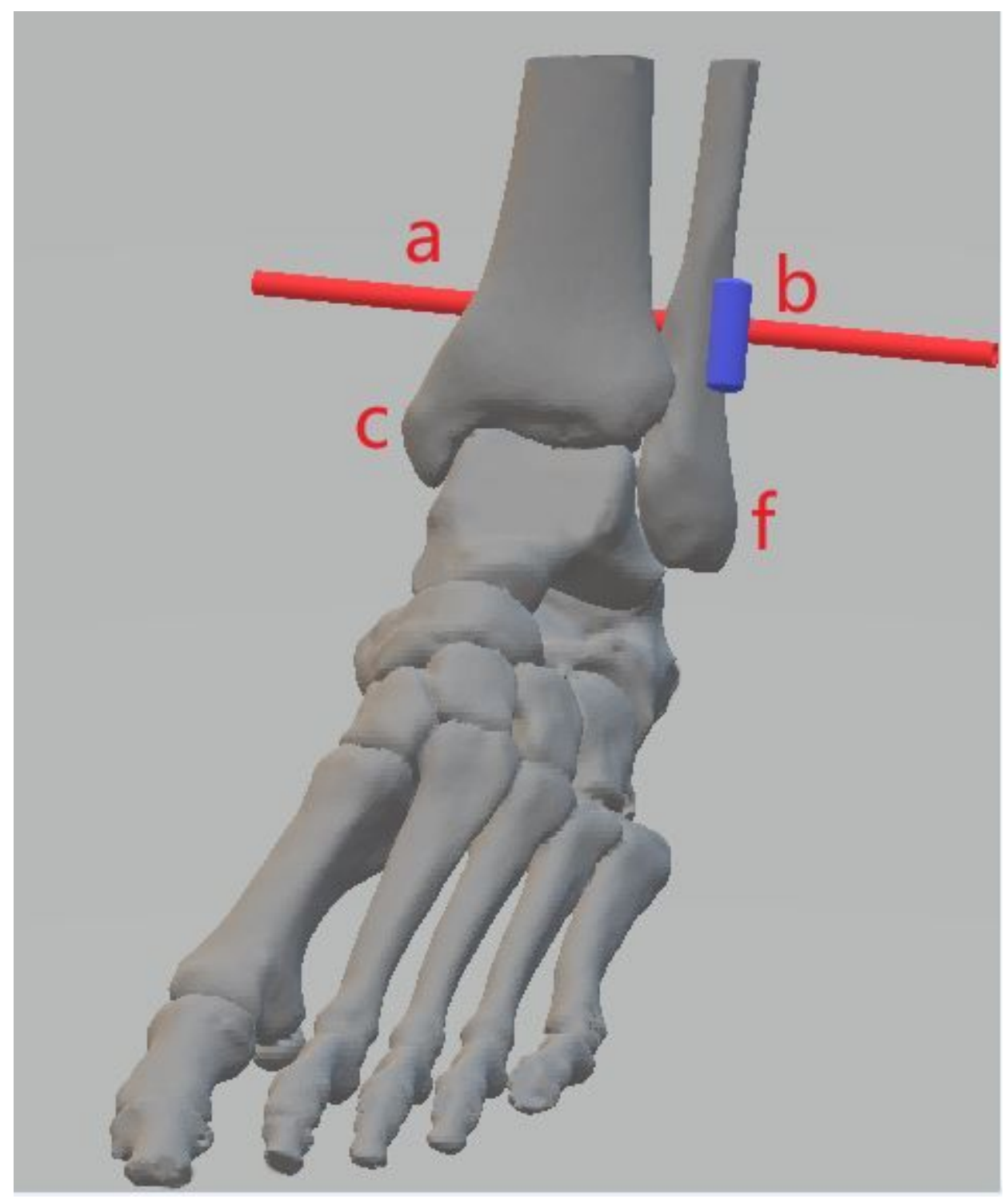

Figure 1

$3 \mathrm{D}$ virtual model. 


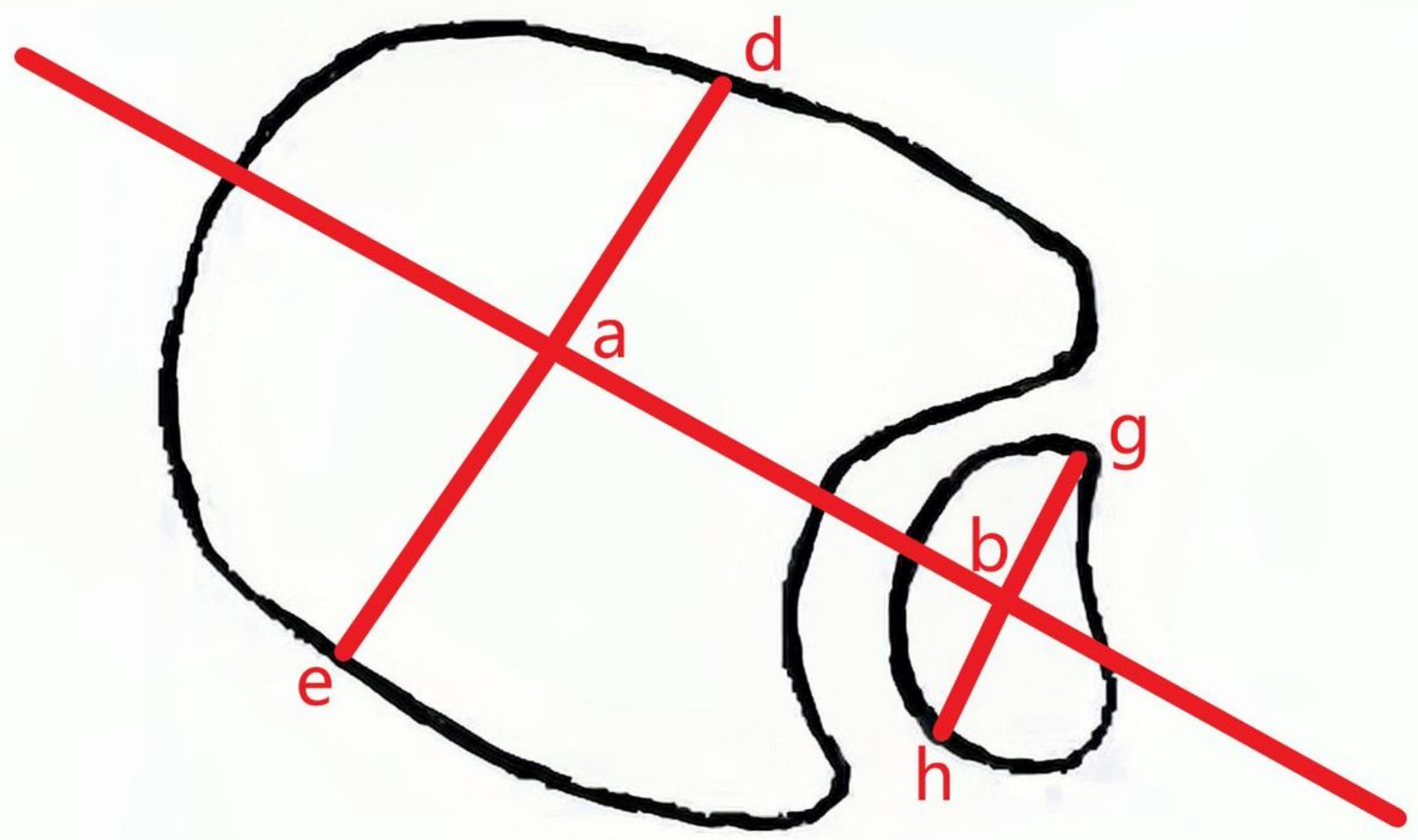

Figure 2

Pattern of tibiofibular surface. 


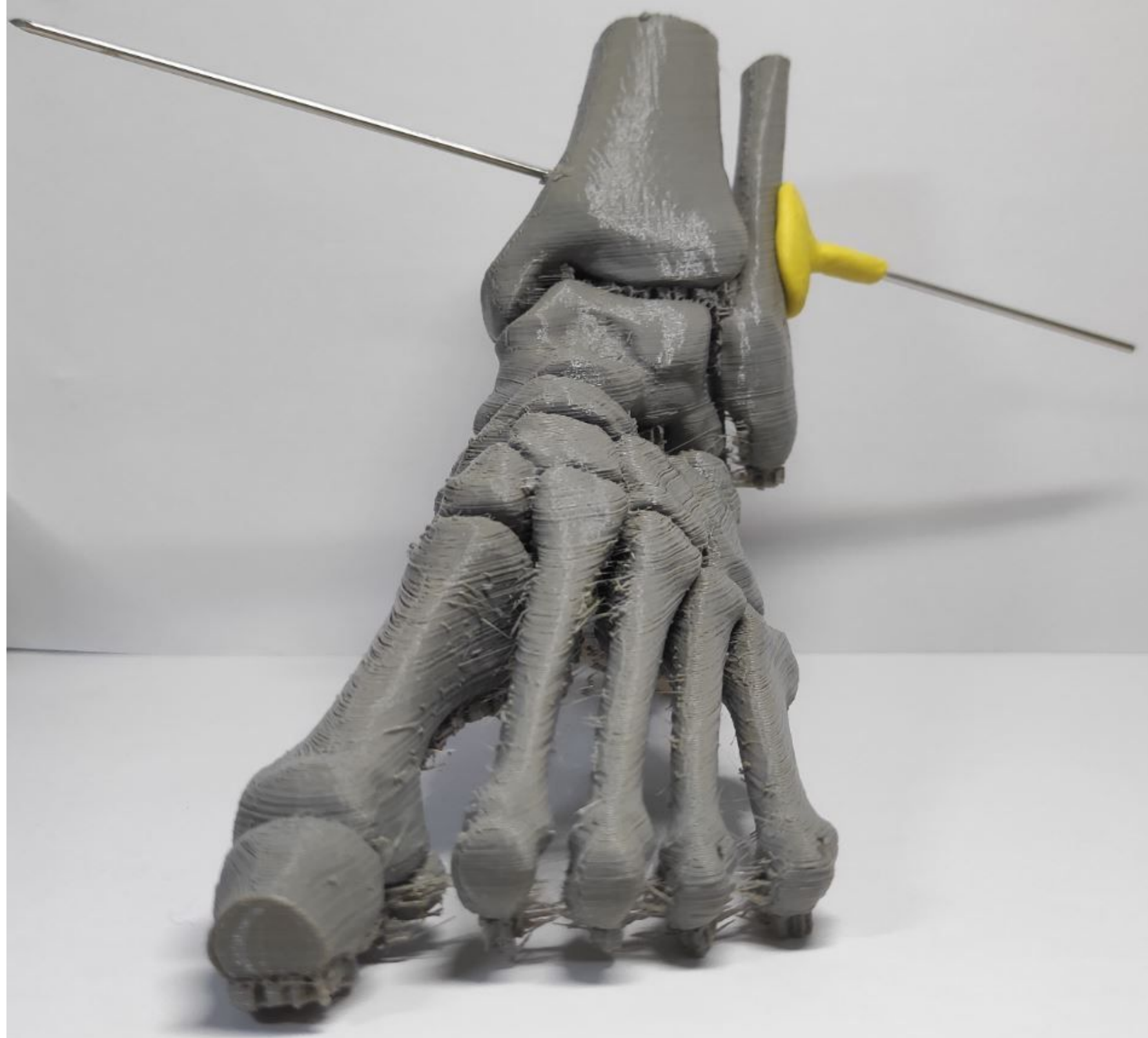

Figure 3

The positive position of actual model. 


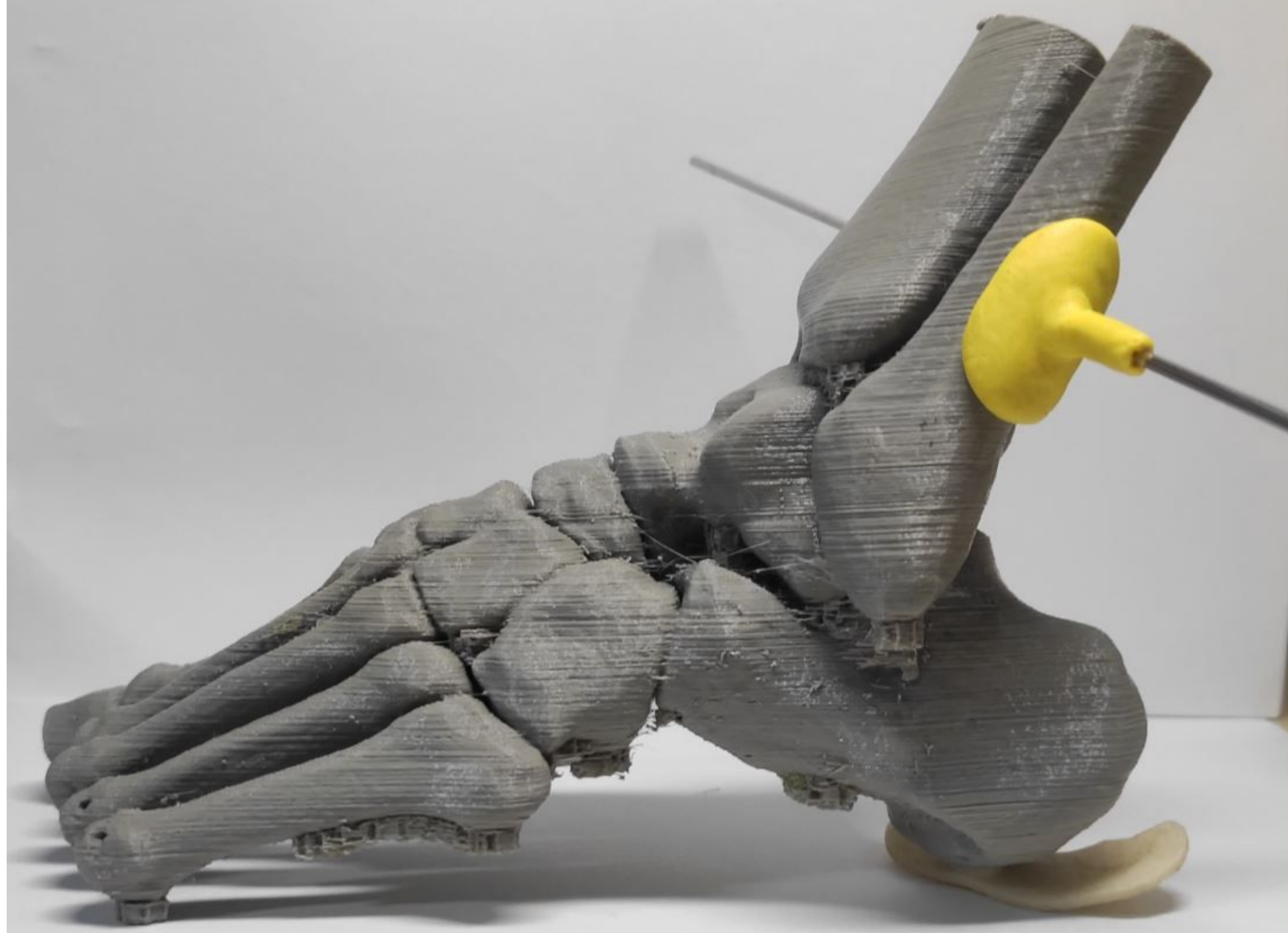

Figure 4

Lateral malleolus position of actual model. 